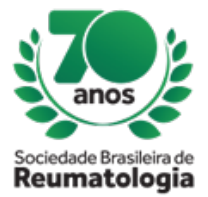

\title{
A CASE OF REFRACTORY PSORIATIC ARTHRITIS WITH EXCELLENT RESPONSE TO THE FIFTH IMMUNOBIOLOGICAL DRUG ATTEMPT
}

Diogo Cunha Lacerda (FAG, Cascavel, PR, Brasil), Lucas Androczevecz Silva (FAG, Cascavel, PR, Brasil), Rafaela Sorpile Araujo (FAG, Cascavel, PR, Brasil), Rodrigo Zuber Maciel (FAG, Cascavel, PR, Brasil), Fabiano de Andrea (FAG, Cascavel, PR, Brasil), Brunno Mehret Moleta (FAG, Cascavel, PR, Brasil), Leandro Silva Pivato (FAG, Cascavel, PR, Brasil), Leonardo Michaelis Schmidt (FAG, Cascavel, PR, Brasil)

\section{BACKGROUND}

Psoriatic arthritis is an inflammatory disease, soronegative for rheumatoid factor (RF), associated to cutaneous psoriasis, which has variable presentations and clinical courses. Nowadays, the treatment normally consists by the use of DMARDs. In refractory situations to the conventional therapy with DMARDs, some other drugs must be considered, like the use of anti-TNF drugs, the use of ustequinumabe (human monoclonal antibody that binds to the IL-12/IL-23 citokynes) and the use of secuquinumabe (antibody against IL-17 citokyne).

\section{CASE REPORT}

Female patient, 44 years old, farmer, diagnosed with psoriasis when she was only 8 months old, started to be affected by arthritis in hands e knees 13 years ago. When she was first biochemically evaluated, inflammatory markers were high, RF was negative and HLAB-27 was positive. Simple radiography showed no sacroiliac involvement. Methotrexate was started 11 years ago, and etanercept was used 3 years ago, resulting in secondary failure (arthritis and cutaneous lesions continued to progress). Considering that, etanercept was exchanged by ustequinumabe (which has a different mechanism of action), but it resulted in a worsening of the cutaneous and joint condition. Therefore, ustequinumabe was exchanged by a third immunobiological drug: infliximab. The clinical response to the induction dose of infliximab was satisfactory, however the disease continued to progress after a while (even after the dose was increased and the space between them was reduced). Then, a fourth drug, adalimumabe, was attempted, bringing out a good cutaneous and joint response in the first 3 months, but with secondary failure after that, by symptoms reactivation. Motivated by the resistance of the condition and the unsuccessful clinical response, opted to switch to another drug which action mechanism different, anti IL-17 (secuquinumabe). The response with this fifth biological was excellent and allowed the patient to remain in sustained remission, presenting PASI 90 and assintomatic after 9 months of the beginning of drug's use.

\section{CONCLUSION}

The way of treating psoriatic arthritis may vary according to presentation and severity that the disease manifests. In cases which the condition is refractory to DMARDs, immunobiological therapy must be considered. In this case, the expected therapeutic response was only reached sustained after fifth drug immunobiological (secuquinumabe). Therefore, the exposed situation exalts that the discrepancy between the clinical response to different immunobiological agents and action mechanism. Thus, this report demonstrates that persistence in the search for therapies with better response to psoriatic arthritis can be extremely beneficial to the pacient. 